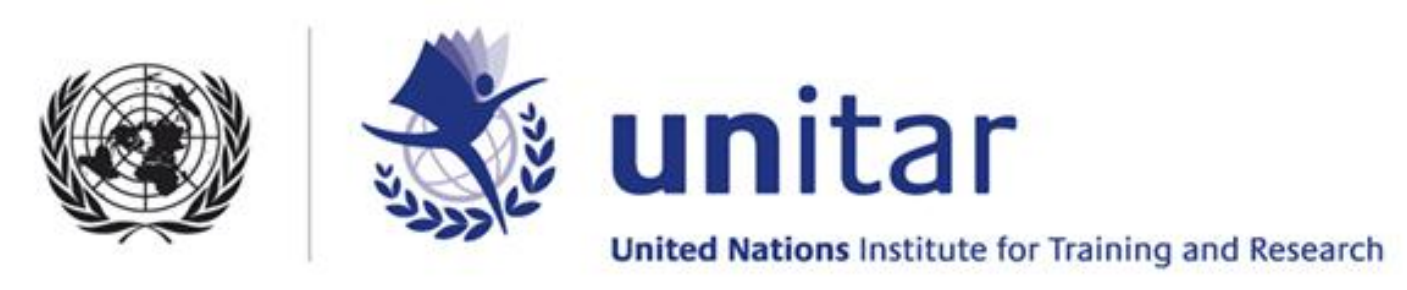

\title{
GRASS GIS in Grid Environment
}

$2^{\text {nd }}$ April 2009

ISGC2009 
Whership, tration, knowledicipe
transfer, action, learning by do
responsiveness, leadership, s

\section{- UNOSAT}

- Grid projects

- GRASS GIS

Integration in Grid environment

Current Status

\section{Future Plans}




\section{What is UNOSAT?}

- UNOSAT is the UN Institute for Training and Research (UNITAR) Operational Satellite Applications Programme, implemented in co-operation with the European Organization for Nuclear Research (CERN).

- Supports early warning, crisis response, human rights, sustainable recovery, vulnerability reduction and local capacity building

\section{What we do?}

- Humanitarian: Rapid mapping in support to disaster management, relief $\&$ human rights

- Training and awareness raising: Customized, from user-perspective

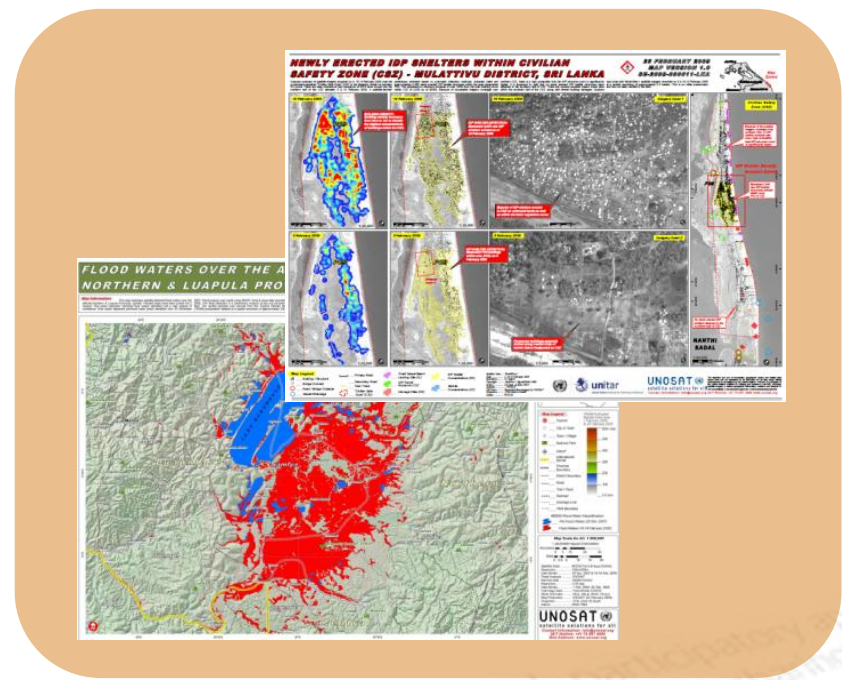




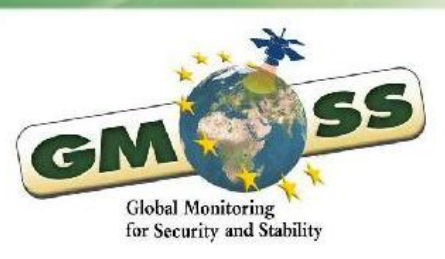

Global Monitoring for Security and Stability March 2004 - February 2008
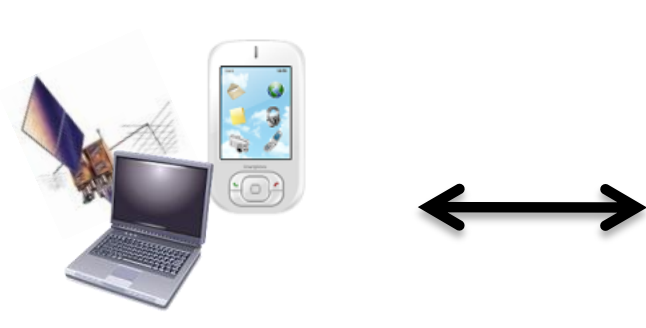

PC based users, Mobile phones with GPS

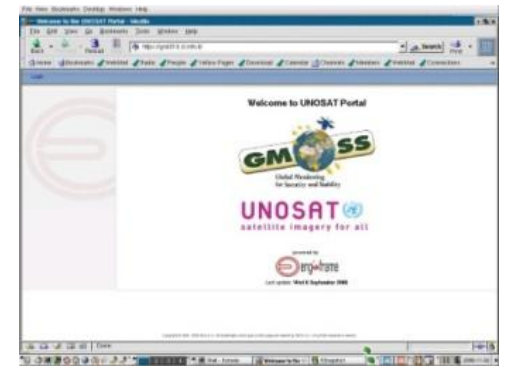

Web-browser based user-interface, mobile phone application

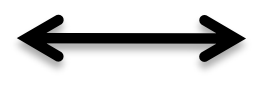

IT Department March 2004 - Present

Satellite imagery storage, metadata processing

-Web interface

-Mobile phone user interface 


\section{Geographic Resources Analysis Support System GRASS GIS}

\section{http://grass.osgeo.org/}

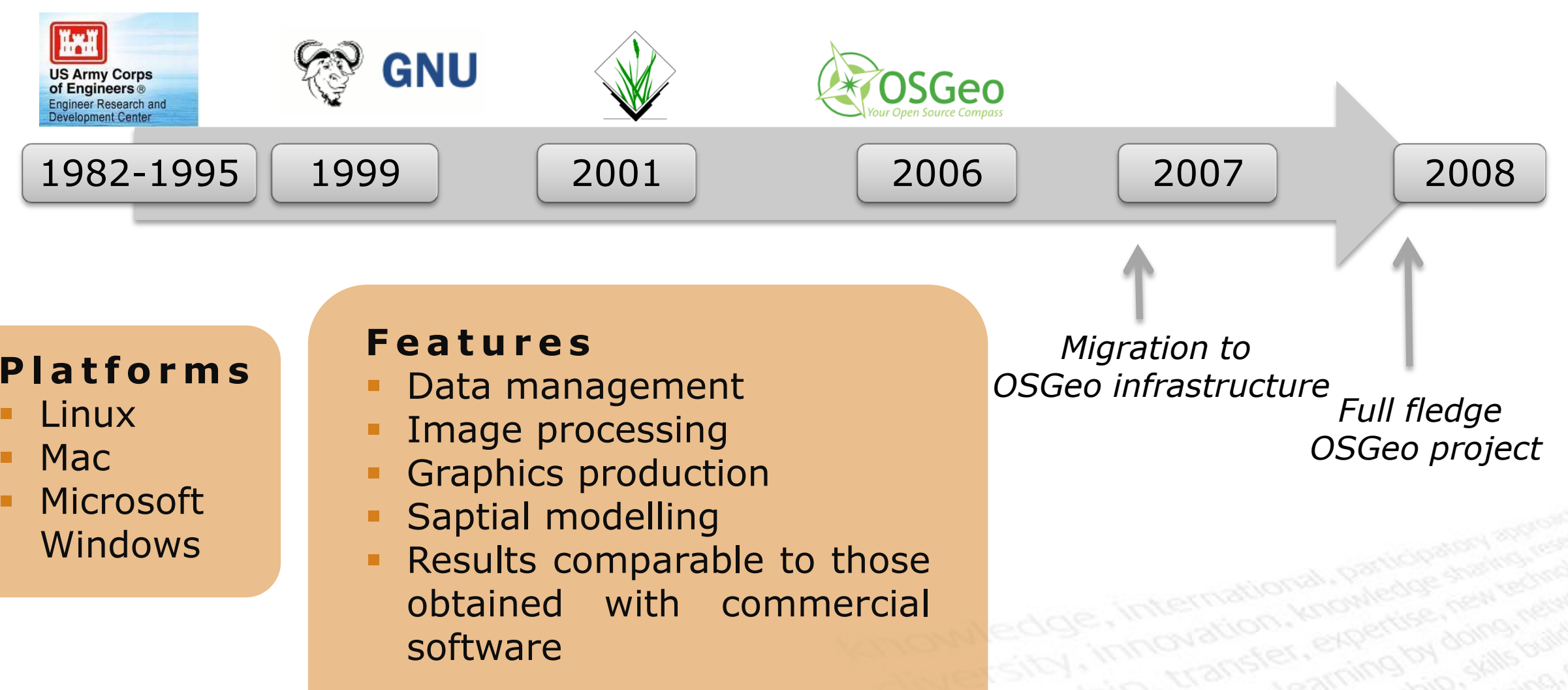


Version: GRASS 5.4.1 released 26 July 2007

Latest stable release: GRASS 6.4.0RC4 released 12 Apr 2009

\section{Aditional Libraries needed for most operations}

GDAL - Geospatial Data Abstraction Library

PROJ.4 - Cartographic Projections Library

GRASS modules are designed under the Unix philosophy and hence can be combined using shell scripting to create more complex or specialized modules by a user without knowledge of C programming.
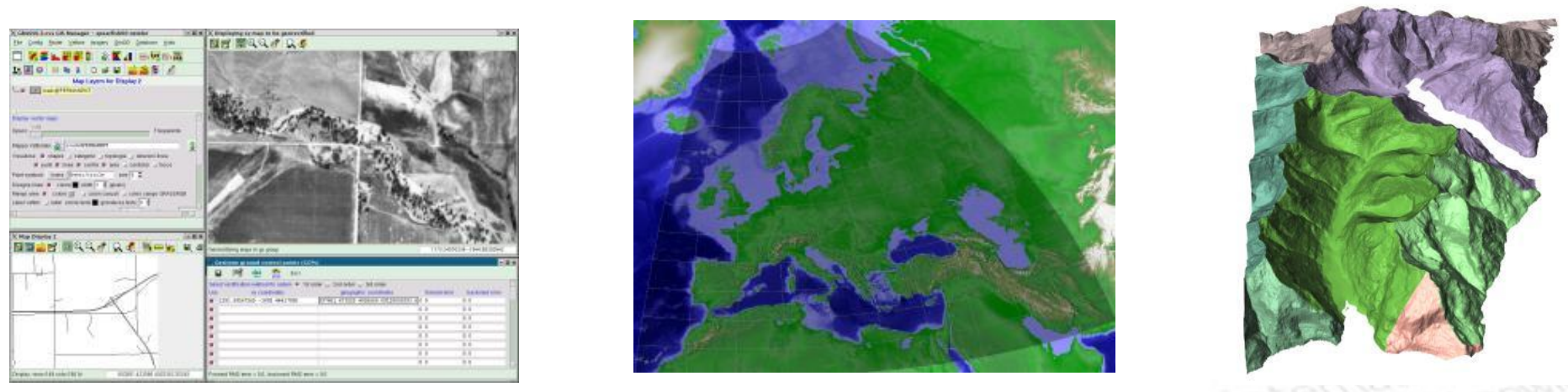


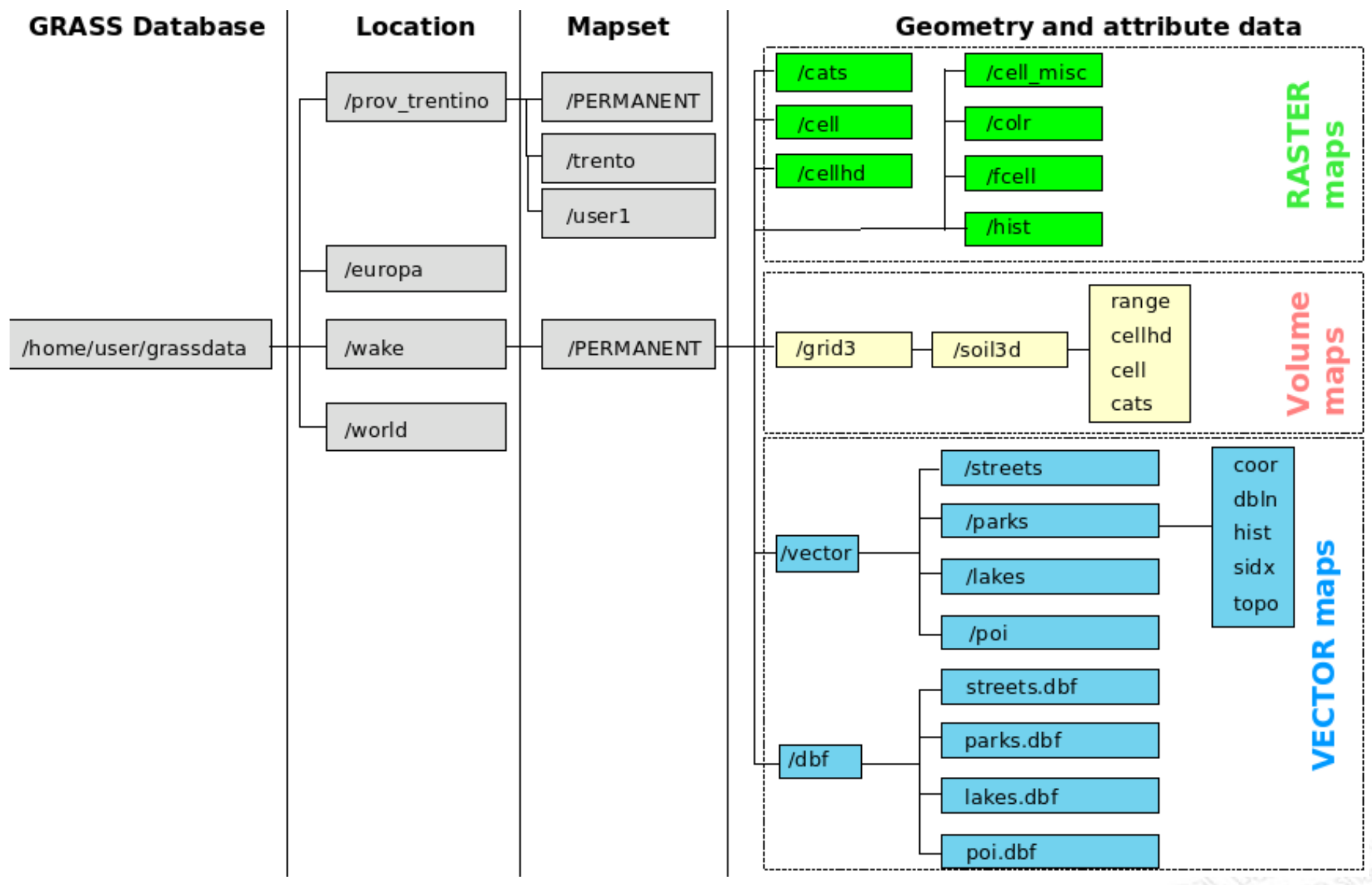




\section{Integration in Grid \\ environment}
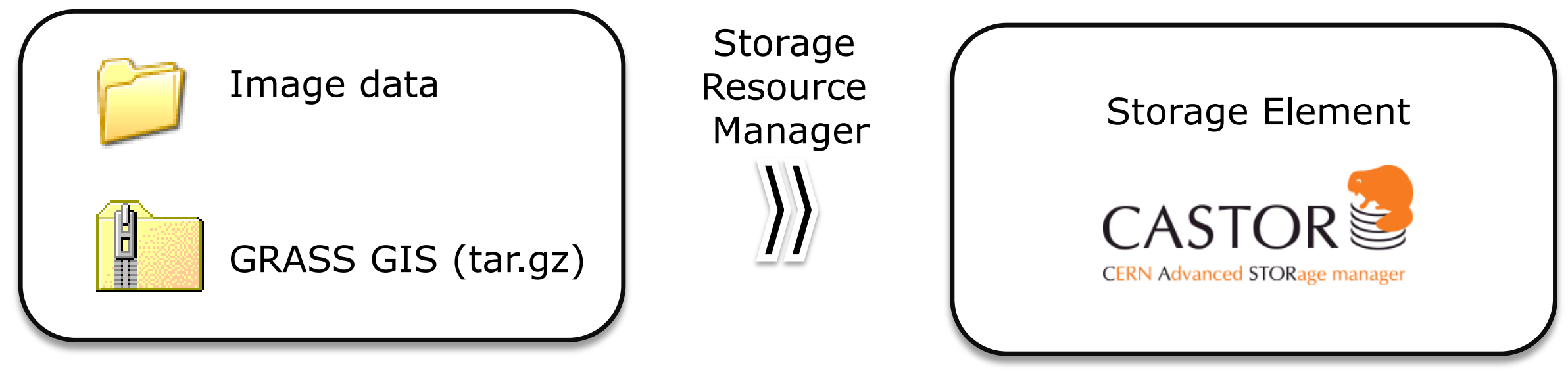

Job Description Language \&

Shell script files
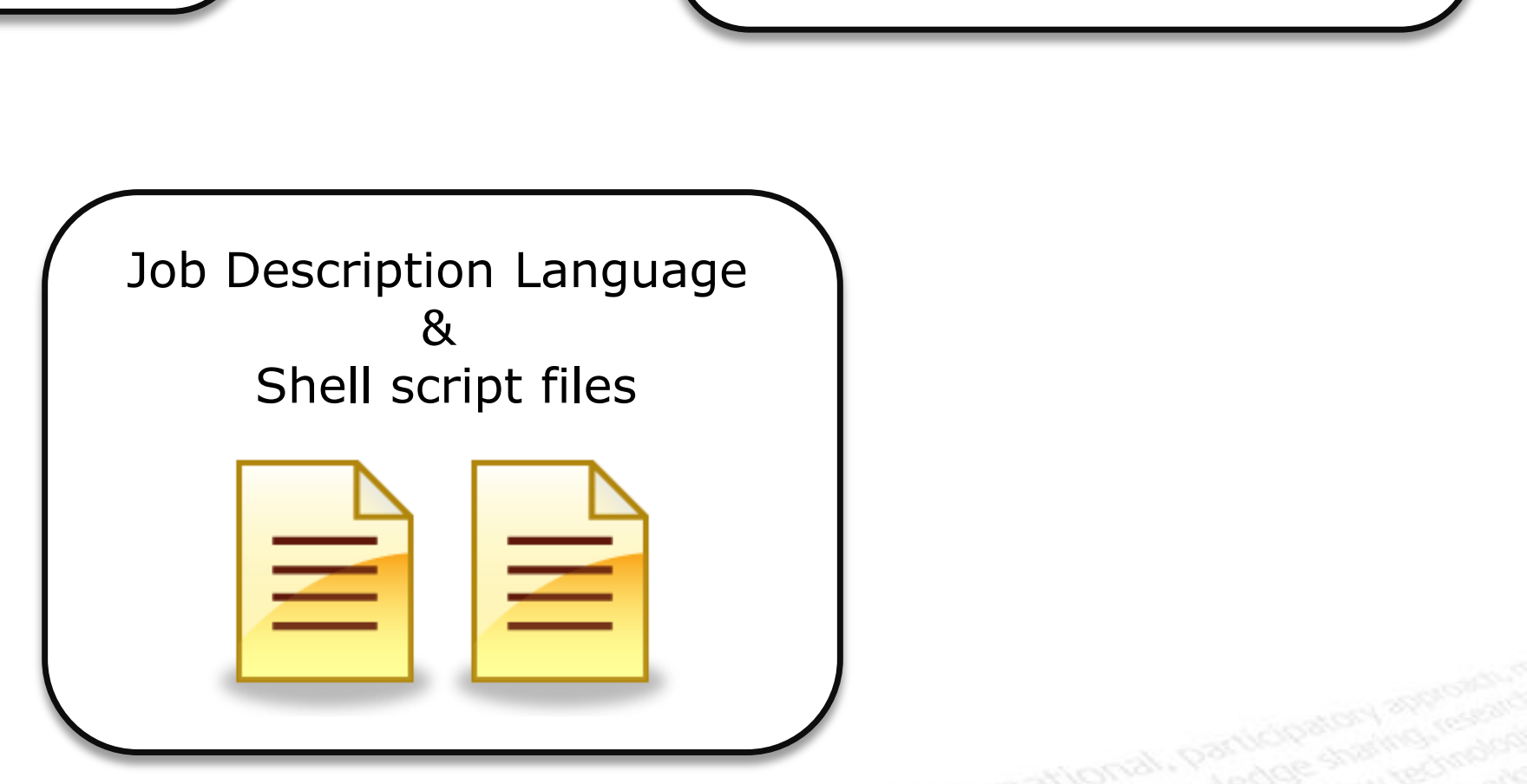


\section{Integration in Grid}

\section{environment}

\section{Image data}

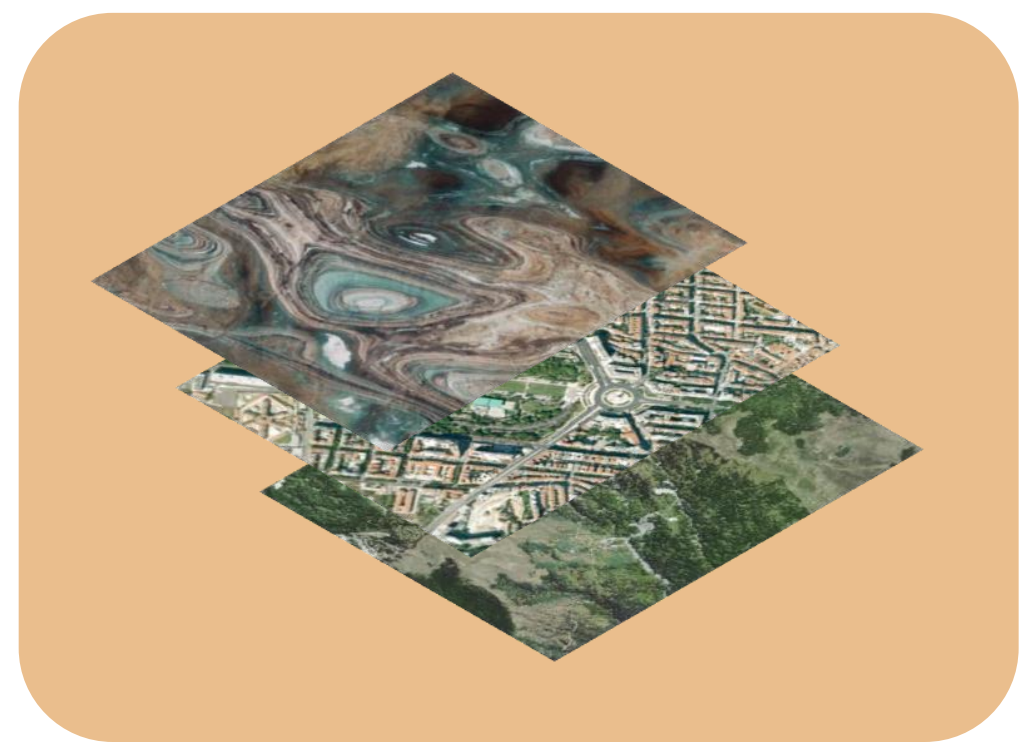

GRASS GIS 5.4.1 Binaries

GDAL - Geospatial Data Abstraction Library

PROJ.4 - Cartographic Projections Library 


\section{Integration in Grid}

Job Description Language \& Shell script files

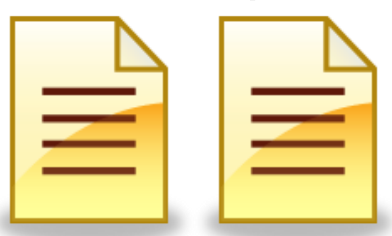

- Very basic jdl file for the inicial testing

- The operations to perform on the data files are defined in the wrapper script which is uploaded at submition time

- To do a diferent analysis of an image we only need to change the wrapper script

\section{filename.jdl}

Executable = "filename.sh";

StdOutput = "filename.out";

StdError = "filename.err";

InputSandbox $=\{" . /$ filename.sh" $\}$;

OutputSandbox $=\{$ "filename.out", "filename.err" $\}$;

filename.sh

Copies the source date and GRASS GIS tar ball to the worker node.

Sets the GRASS GIS environment variables

Executes the GRASS commands

Copies the results back to the storage element 


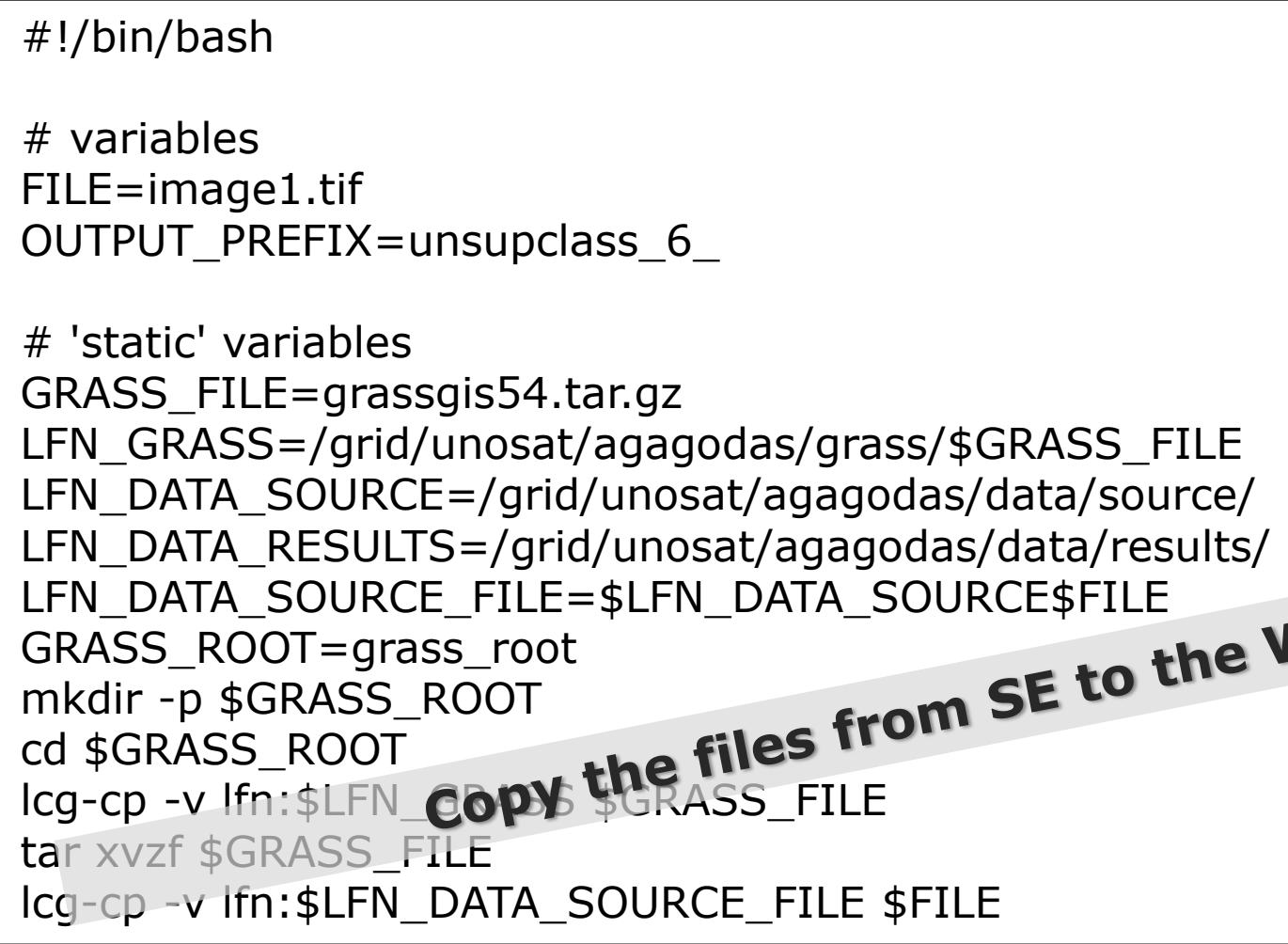


grassdata $=\$($ Is $\$$ GISDBASE $/$ location_name $/$ MAPSET $/$ cell/ $)$

i. group group=group subgroup=subgroup inputäands

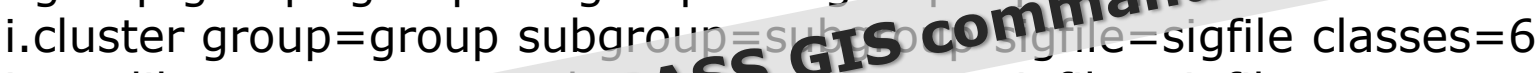

i. maxlik group=groun su GRASS Subgroup sigfile=sigfile

class=unsupclass ExeC

r.out.tiff input=unsupclass output=unsuplcass_\$(date

+"\%d\%m\%y_\%H\%M\%S")

for outputfile in 'Is \$OUTPUT_PREFIX*'; do echo "\#\#\# Saving output file

\$outputfile:"; Icg-cr -v file:\$outputfile -d silfs to SE

public.cern.ch/castor/cern.ch\$COPy results LLTS\$outputfile -I

\$LFN_DATA_RESULTS\$output CO, done 
- Data as been manually uploaded to the Storage Element

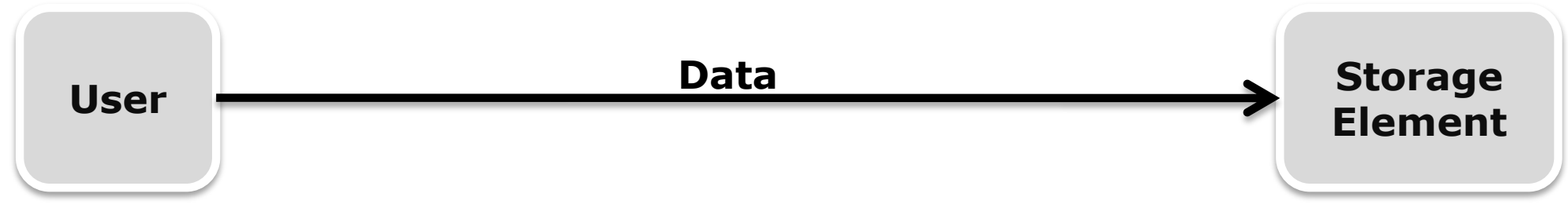

- A generic self contained GRASS GIS instalation has been created

- Two different types of jobs have been sucessfully submited and executed.

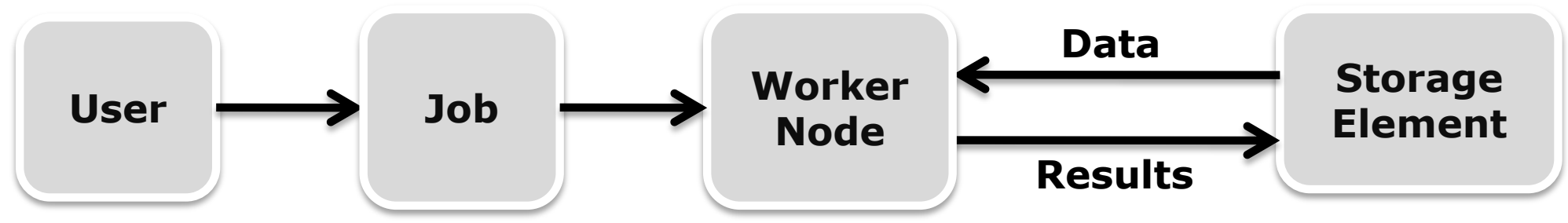

- The results have been manually retrieve from the Storage Element

- The validity of the results have been verified

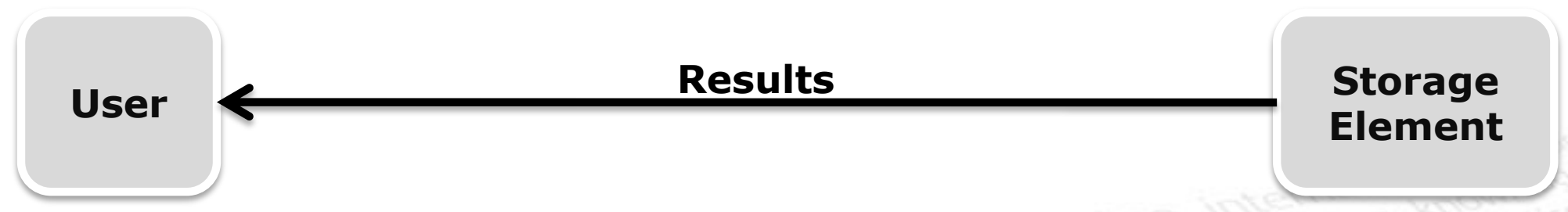


- Upload all available imagery to the storage element while registering it in a metadata database.

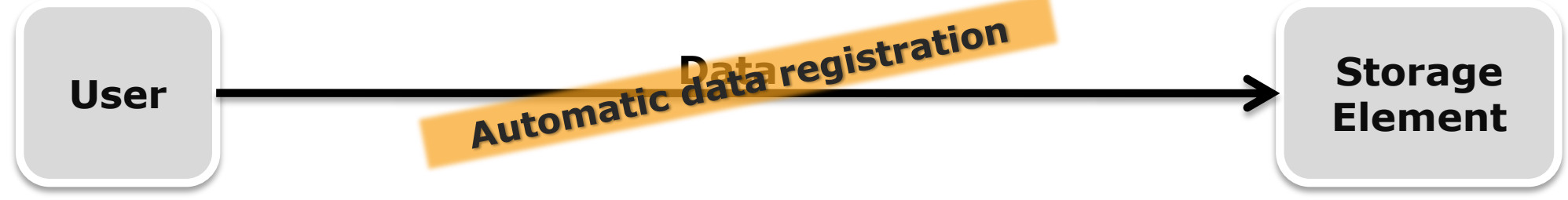

- Select and test a list of predifined image processing operations.

- Create a web portal were registered users can request that existing images are processed using GRASS GIS. This will mask the use of the Grid infraestructure from the users and enable the use of the system from different platforms.

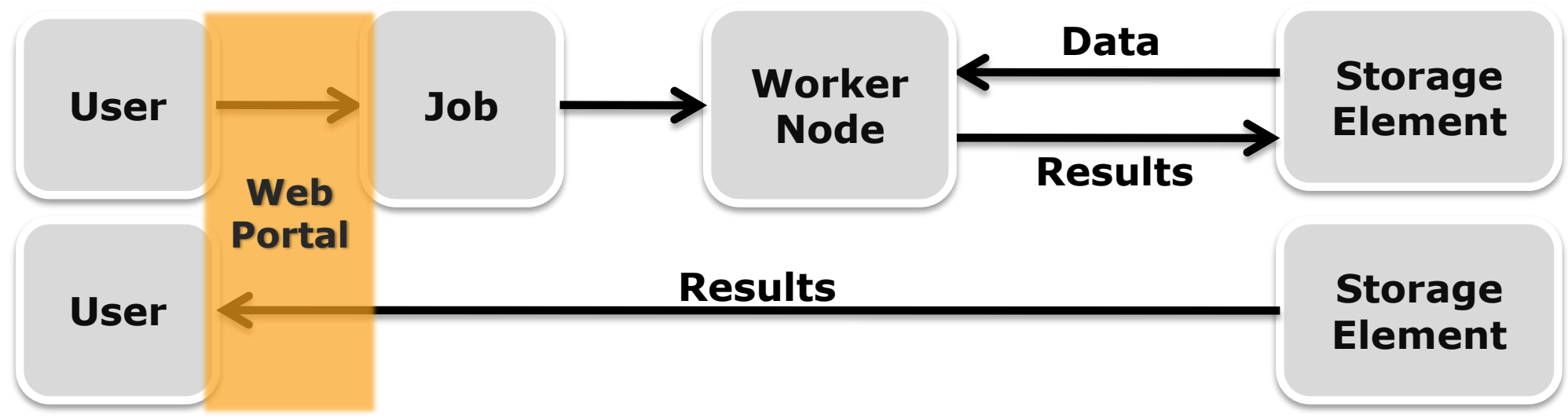

- Perform common operations on all available imagery (extract contour lines, unsupervised classification, vegetation index) and vector data. 


\section{ww w. unitar.org/unos a \\ unos a $\mathrm{t}$ @ u n i t a r.org}

Th a n k y o u !

United Nations Institute for Training and Research
Institut des Nations Unies pour la Formation et la Recherche Instituto de las Naciones Unidas para Formación Profesional e Investigaciones

Учебный и научно-исследовательский институт Организации Объединенных Наций unitar

Palais des Nations

CH-1211 - Geneva 10

Switzerland

$\mathbf{T}+41-22-917-8455$

$F+41-22-917-8047$

联合国训练形究所 WWW.unitar.org 